\title{
A AVALIAÇÃO DA VINCULAÇÃO DO ADULTO: UMA REVISÃO CRÍTICA A PROPÓSITO DA APLICAÇÃO DA ADULT ATTACHMENT SCALE-R (AAS-R) NA POPULAÇÃO PORTUGUESA
}

\author{
M. Cristina Canavarro ${ }^{1}$ \\ Pedro Dias ${ }^{2}$ \\ Vânia Lima ${ }^{3}$
}

\begin{abstract}
Resumo: O presente artigo, numa primeira parte, procura esclarecer algumas questões conceptuais em torno do conceito de vinculação do adulto. Seguidamente são referidas algumas implicações dos tópicos abordados para a avaliação da vinculação do adulto, assim como debatidos alguns aspectos mais polémicos associados às metodologias utilizadas.

Por último, focando a avaliação da vinculação do adulto através de questionários de auto-resposta, apresentam-se alguns dos estudos psicométricos realizados em Portugal com a Adult Attachment Scale-R (Collins \& Read, 1990), que na versão portuguesa recebe a designação de Escala de Vinculação do Adulto (EVA).
\end{abstract}

Palavras-chave: vinculação; vinculação do adulto; avaliação; Adult Attachment Scale, Escala de Vinculação do Adulto

The Assessment of Adult Attachment: A critical review on the sequence of the application of the Adult Attachment Scale-R $(A A S-R)$ to the Portuguese population (Abstract): This article, in its first part, tries to gather knowledge about some of the conceptual issues on adult attachment. These conceptual issues are discussed in terms of the adult attachment assessment and also some of the most polemical aspects on methodology are also highlighted.

Finally, focusing on the assessment of adult attachment through self-report questionnaires, some psychometric data and results of Portuguese studies concerning

\footnotetext{
${ }^{1}$ Faculdade de Psicologia e de Ciências da Educação da Universidade de Coimbra. Toda a correspondência sobre este artigo deve ser enviada para Faculdade de Psicologia e de Ciências da Educação da Universidade de Coimbra, Rua do Colégio Novo, Apartado 6153,3001-802 Coimbra, ou para mccanavarro@fpce.uc.pt.

2 Instituto de Educação, Universidade Católica Portuguesa do Porto. Bolseiro de doutoramento pela FCT (ref. SFRH/BD/6944/2001).

${ }^{3}$ Departamento de Psicologia, Universidade do Minho. Bolseira de doutoramento pela FCT (ref. SFRH/BD/13291/2003).
} 
the adaptation of Adult Attachment Scale-R (Collins \& Read, 1990), named "Escala de Vinculação do Adulto" (EVA) are presented.

Key-words: attachment; adult attachment; assessment; Adult Attachment Scale

\section{Introdução}

Embora Bowlby, ao longo da sua obra $(1973,1977,1988)$, reconheça, em diversas ocasiões, a importância da vinculação ao longo de todo o ciclo de vida do ser humano, a sua investigação centrou-se fundamentalmente na infância. Bowlby e Ainsworth focaram a atenção nas origens desenvolvimentais do sistema de vinculação, centrando-se na vinculação da criança aos pais e, sobretudo, à mãe.

A partir da década de 80 , um conjunto de investigadores ofereceu contributos distintos que tornaram relevante o estudo da vinculação durante a adolescência e idade adulta. Entre eles, salientam-se os estudos de Main e da sua equipa sobre a dimensão representacional da vinculação, dos quais resultou a construção da Adult Attachment Interview (AAI, George, Kaplan \& Main, 1984), os trabalhos de Hazan e Shaver sobre a aplicação da classificação da vinculação de Ainsworth à organização emocional e comportamental dos adolescentes e jovens adultos, explorando o amor romântico enquanto processo de vinculação e, ainda, os trabalhos de um conjunto de investigadores que, de forma separada, desenvolveram instrumentos para avaliar aspectos diversos relacionados com a vinculação.

O último tipo de contribuição mencionado, a construção de instrumentos de avaliação dirigidos a adolescentes e a adultos, a maior parte de auto-resposta (oferecendo alguns deles, de forma associada, também o formato de entrevista), tem proliferado e interessa-nos particularmente, no âmbito do presente artigo, motivo pelo qual desenvolveremos mais detalhadamente este tópico no ponto dedicado às questões sobre a avaliação da vinculação do adulto.

Os contributos que anteriormente referimos e, especificamente, a possibilidade de avaliar domínios da vinculação do adulto, permitiram que a Teoria da Vinculação tenha vindo a ser utilizada por um número crescente de investigadores, como quadro conceptual de referência, ao estudar aspectos psicológicos diversos relativos à idade adulta.

Mais especificamente, encontramos vários trabalhos que, com referência à Teoria da Vinculação, estudam processos de continuidade e mudança ao longo da vida (Fraley, 1999; Scharfe \& Bartholomew, 1994; Mena-Matos, 2002), avaliam o amor romântico como um processo de vinculação (e.g., Hazan \& Shaver, 1987), testam a influência das relações estabelecidas 
com os pais na infância e adolescência nas relações estabelecidas na idade adulta (e.g., van IJzendoorn, 1995) e procuram compreender o papel da vinculação nas estratégias de coping associadas aos pedidos de ajuda (Bartholomew, Rebecca, Cobb \& Poole, 1997; Fraley \& Shaver, 1998).

Recentemente, é ainda frequente encontrarmos a Teoria da Vinculação utilizada como grelha conceptual na investigação sobre questões mais particulares da idade adulta, tais como a violência conjugal (Holtzworth-Munroe, Stuart \& Hutchinson, 1997); os maus-tratos infantis, a orientação sexual (Kurdek, 2002), a aliança terapêutica (Dozier, 1990; Mallinckrodt, Cocle \& Gantt, 1995) e diversos quadros clínicos psicopatológicos (e.g., Canavarro, 1999; Carlson \& Sroufe, 1995; Dozier, 1990; Eng, Heimberg, Hart, Schneier \& Liebowitz, 2001).

Constatamos, assim, que a Teoria da Vinculação tem permitido o desenvolvimento de uma das linhas de investigação mais abrangentes, profundas e criativas da nossa era (Cassidy \& Shaver, 1999). Por esta razão, torna-se ainda mais premente o debate e esclarecimento de questões conceptuais e metodológicas associadas ao processo de avaliação da vinculação na idade adulta. $\mathrm{O}$ presente artigo pretende ser, por isso, uma contribuição para esta clarificação. Centrando-nos na avaliação do adulto através de questionários de auto-avaliação, apresentamos alguns dos estudos psicométricos resultantes da aplicação da Adult Attachment Scale-R (Collins $\&$ Read, 1990) à população portuguesa.

\section{A vinculação do adulto: questões conceptuais}

De acordo com Crowell, Fraley e Shaver (1999), o conceito de vinculação do adulto pressupõe duas ideias fundamentais, com importantes implicações para os processos de avaliação. A primeira prende-se com a assunção sobre os aspectos normativos do sistema de vinculação e com a sua relevância durante a idade adulta; a segunda, com a presença de diferenças individuais na organização da vinculação, no contexto das relações interpessoais. Os tópicos mencionados parecem-nos, de facto, centrais para o esclarecimento do tema e iremos utilizá-los como guia no esclarecimento das questões conceptuais sobre a vinculação na idade adulta, enriquecendo-os com aspectos que entendemos como associados e que permitem aprofundar o conceito de vinculação do adulto.

\section{A importância da vinculação ao longo da vida}

Bowlby $(1973,1980)$ propôs que um sistema comportamental está subjacente à tendência das crianças para formar fortes laços emocionais 
com os seus cuidadores, as suas figuras de vinculação. Este sistema de vinculação, quando activado, permite à criança procurar a proximidade física com a figura de vinculação e, assim, contribui para a promoção de segurança.

Embora o sistema de vinculação esteja permanentemente activo, funcionando no que podemos designar num nível mínimo, permitindo a constante monitorização das situações ambientais e da disponibilidade da figura de vinculação, os comportamentos de vinculação só são activados em alturas de stress, perigo ou situações novas. A sua activação permite a manutenção da proximidade e contacto com a figura de vinculação, existindo, desta forma, uma especificidade das manifestações da vinculação relativamente ao contexto. Quando a criança percepciona a figura de vinculação como disponível e o ambiente como tranquilo, pode explorar o meio com confiança, fenómeno de interacção que Ainsworth, Blehar, Waters e Wall (1978) designaram por base segura.

$\mathrm{Na}$ linha do sugerido por West e Sheldon-Keller (1994), parece-nos importante para o esclarecimento do conceito de vinculação do adulto: (1) encontrar elementos de congruência entre a vinculação durante a infância e a que sucede em épocas posteriores do ciclo de vida; (2) reconhecer as diferenças entre os processos que sucedem nas épocas diferentes da vida e (3) distinguir as relações de vinculação na idade adulta de outras estabelecidas nesse período.

Sobre a questão da congruência da vinculação ao longo da vida, já Bowlby (1969/1982) tinha considerado que a vinculação na idade adulta é semelhante, na sua natureza, à que ocorre durante a infância e apontou poucas diferenças entre as relações estabelecidas entre as crianças e as figuras cuidadoras e as relações formadas entre pares ou companheiros românticos.

A este propósito, Ainsworth (1991) considerou o fenómeno de base segura como o elemento central da vinculação ao longo da vida, referindo que uma relação de vinculação segura é aquela que facilita o funcionamento e competência fora da relação.

Como elementos de congruência entre a vinculação na infância e na idade adulta, Weiss (1982/1991) apontou a similaridade das características emocionais e comportamentais (e.g., desejo de proximidade à figura de vinculação em alturas adversas, conforto na presença da figura de vinculação, ansiedade face à inacessibilidade da figura de vinculação, respostas de luto em situação de perda), a generalização da experiência, dado que elementos emocionais associados à vinculação durante a infância são expressos nas relações de vinculação na idade adulta, e, ainda, a ligação temporal entre os fenómenos, uma vez que a centralidade dos pares como figuras de 
vinculação se encontra associada ao esbatimento dos progenitores enquanto figuras de vinculação primárias.

Para diferenciar a vinculação estabelecida na infância e na idade adulta, Weiss (1982) refere que as relações de vinculação na idade adulta são tipicamente estabelecidas entre pares, o sistema comportamental implicado não se destaca tanto de outros semelhantes, dada não estar em causa, na maioria das vezes, a sobrevivência e, por último, o autor aponta a sua inclusão em relações que muitas vezes comportam dimensões de envolvimento sexual.

Hinde e Stevenson-Hinde (1986) apontam também como distintivo da vinculação característica da infância e da idade adulta o facto de serem necessários acontecimentos indutores de stress mais fortes para activar o sistema de vinculação, devido ao desenvolvimento da capacidade de representação, que permite fixar a figura de vinculação e a maior destreza para lidar, de forma autónoma, com pequenas adversidades do dia-a-dia.

No entanto, a diferença mais referida na literatura, apontada por muitos autores (Crowell et al., 1999; Hinde, 1997; Hinde \& Stevenson-Hinde, 1986, Weiss, 1982) como a mais significativa, prende-se com a natureza recíproca das relações de vinculação estabelecidas na idade adulta, por comparação com a natureza complementar das relações de vinculação estabelecidas na infância. Por outras palavras, nas relações de vinculação entre adultos, prestam-se e recebem-se cuidados alternadamente, de acordo com o contexto e necessidades de cada interveniente na relação.

Ao longo do tempo, diversos autores têm, também, procurado distinguir as relações de vinculação na idade adulta de outras estabelecidas na mesma época do ciclo de vida, salientando sempre a sua função única de promover uma sensação de segurança e pertença. Neste sentido, as relações de vinculação na idade adulta distinguem-se das de afiliação (Weiss, 1982; West \& Sheldon-Keller, 1994), dependência (Ainsworth, 1972; Rutter, 1995) e das que comportam envolvimento sexual (Berman \& Sperling, 1994; West \& Sheldon-Keller, 1994).

\section{Diferenças individuais e modelos internos dinâmicos}

A ideia da existência de diferenças individuais na organização da vinculação emergiu do trabalho de Ainsworth et al. (1978). As diferenças encontradas entre os diversos padrões de vinculação na infância parecem desenvolver-se primariamente de acordo com as diferentes experiências de interacção com a figura de vinculação.

Desta forma, um padrão "seguro" estaria associado a uma interacção caracterizada por protecção e conforto por parte da figura de vinculação em ocasiões adversas para a criança. Em alturas oportunas, o suporte e dispo- 
nibilidade da figura de vinculação optimizariam o comportamento de exploração da criança. Por sua vez, os padrões "inseguros" ("evitante" e "ambivalente") emergiriam em contextos de interacção em que o comportamento de vinculação da criança fosse seguido, respectivamente, de rejeição ou inconsistência, por parte da figura de vinculação. Para reduzir a ansiedade gerada por estas situações, a criança desenvolveria estrategicamente respostas de hipo e hiperactivação do sistema de vinculação, consideradas assim adaptativas ao contexto, mas desadequadas fora do âmbito desta relação particular, dado que comprometem o sistema de exploração.

Muitos dos desenvolvimentos ocorridos no âmbito da teoria e investigação na área da vinculação do adulto têm subjacente a proposta formulada por Bowlby (1973/1980), segundo a qual as crianças desenvolveriam um conjunto de expectativas acerca de si próprias, dos outros e do mundo, que designou por modelos representacionais ou modelos internos dinâmicos. Estes modelos permitiriam, numa fase inicial, interpretar e prever o comportamento da figura de vinculação e, ao longo da vida, seriam utilizados como guias comportamentais, constituindo uma base para interpretação de experiências relacionais.

Como referimos anteriormente, as diferenças individuais na organização da vinculação emergiriam na sequência do contexto de interacção entre a criança e a figura de vinculação, que possibilitaria a construção dos modelos internos dinâmicos. Embora estes modelos sejam relativamente estáveis e possam operar automaticamente e influenciar expectativas, estratégias e comportamentos nas relações estabelecidas posteriormente (Bretherton, 1985), foram adjectivados de dinâmicos ("working" models, no original). A opção por esta designação enfatiza a possibilidade de serem reformulados no contexto de experiências de vinculação significativas, capazes de infirmarem experiências anteriores e/ou de possibilitarem novas concepções sobre as experiências passadas, abrindo caminho à possibilidade de mudança ao longo da vida.

As diversas concepções sobre a origem das diferenças individuais na organização da vinculação estão associadas à discussão em torno da estabilidade e da mudança da vinculação, ao longo do ciclo de vida, e comportam implicações para a avaliação da vinculação na idade adulta.

\section{Abordagens conceptuais da vinculação do adulto}

Na literatura, a vinculação do adulto surge conceptualizada de três formas distintas (Berman \& Sperling, 1994; Shaver \& MiKulincer, 2000): (1) vinculação como estado, que emerge em situações de stress, num esforço para restabelecer contacto com a figura de vinculação; (2) vinculação como traço ou tendência para formar relações de vinculação similares ao 
longo da vida; (3) vinculação como um processo de interacção no contexto de uma relação específica.

A vinculação como estado remete para os primeiros trabalhos de Bowlby (1973) de caracterização das respostas das crianças face à separação da mãe, dos quais resultou a identificação de diversas fases: "protesto", "desespero e desorganização" e "desvinculação"4. A constatação de que os adolescentes e adultos, em situações de separação da figura de vinculação (como acontece nos casos de saída de casa dos pais, separação conjugal ou morte de uma figurara de vinculação), exibiriam respostas semelhantes às observadas nas crianças separadas da figura de vinculação levou a comcluir-se que estes comportamentos seriam "reacções normativas de intolerância à inacessibilidade da figura de vinculação" (Weiss, p. 135).

Nas investigações mais recentes, a forma mais comum de conceptualizar a vinculação do adulto prende-se com a assunção da existência de diferenças individuais estáveis ao longo do tempo. Subjaz a esta concepção a ideia de que modelos internos dinâmicos específicos determinam as respostas às separações e reuniões, reais ou imaginadas, da figura de vinculação. Estes modelos representacionais são entendidos como consistentes ao longo do tempo e dos diversos contextos relacionais e, para a maioria dos autores, como já mencionámos, encontram as suas raízes nas experiências de vinculação precoces. A ligação entre os modelos internos dinâmicos da infância e os da idade adulta não está completamente esclarecida e continua a ser tema de debate. Há um consenso geral sobre a estabilidade da vinculação durante a idade adulta, no entanto, esse padrão pode não corresponder ao observado na infância (Shaver et al., 1988). A este propósito, como referimos, alguns autores (e.g., Main et al., 1985) têm proposto mecanismos explicativos da mudança na organização da vinculação ao longo do tempo. Adicionalmente, também, continua por esclarecer a contribuição das diferentes relações de vinculação na infância para a construção de uma estratégia organizada do sistema de vinculação (Lewis, 1994; Howes, 1999) e a sua associação com a vinculação na idade adulta.

O número de estilos de vinculação dos adultos também não é consensual. De forma geral, a maior parte dos autores propõe a existência de apenas

\footnotetext{
${ }^{4}$ A referir que a descrição das fases inicialmente realizada por Bowlby sofreu importantes reformulações (Bowlby, 1980) após a observação clínica e o trabalho de investigação levado a cabo com adultos que tinham sofrido perdas. A constatação de que, muitas vezes, os indivíduos após a perda negam-na, possivelmente por esta ser muito dolorosa e incompreensível, levou à introdução de uma fase inicial designada por "choque e negação". Também com base na capacidade dos adultos para lidar cognitiva e emocionalmente com a perda e apoiado na evidência de que a reunião com uma figura de vinculação pode provocar elevada activação do sistema de vinculação, Bowlby alterou a designação da última fase mencionada de "desvinculação" para "reorganização".
} 
um estilo seguro e de vários inseguros. A revisão da literatura permite-nos referir investigadores que propõe a existência de dois, de três e mesmo de quatro estilos de vinculação insegura. Destacamos os mais referidos.

O modelo original de Ainsworth et al. (1978), que inclui os estilos "seguro", "evitante" e "ansioso/ambivalente" foi adoptado por Hazen e Shaver (1987) para estudar as relações heterossexuais como processos de vinculação. A equipa de Main também adoptou inicialmente um modelo tripartido, onde mais tarde incluiu uma quarta categoria: "autónomo", "desligado", "preocupado" e a classificação suplementar "unresolved" (Main et al., 1985), habitualmente associada a perdas ou situações traumáticas.

Com base nas representações internas sobre si próprio e sobre os outros, Bartholomew e Horowitz (1991) propuseram um modelo com quatro categorias. Para além das dimensões "segura" e "preocupada", contempladas nos outros modelos até à data, os autores identificam dois subtipos de estilo "evitante": o "evitante-desligado", que combina a percepção do próprio como merecedor de cuidados dos outros com a representação destes como não respondendo às suas necessidades; e o "evitante-amedrontado", que se caracteriza pela percepção do próprio como não merecedor dos cuidados dos outros, combinado com uma avaliação destes como pessoas em quem não se pode confiar.

Já Bowlby (1980) inicialmente tinha proposto algumas variações da insegurança da vinculação do adulto com origens na infância, incluindo os estilos "prestação de cuidados compulsiva", "procura de cuidados compulsiva", "autoconfiança compulsiva" e "desvinculação emocional".

Para além das variações que acabámos de referir relativas ao número de estilos considerados por diversos autores, encontramos também diversidade em relação às dimensões consideradas como subjacentes aos estilos referidos e, ainda, disparidade em relação à natureza dos modelos considerados, que incluem abordagens categoriais, dimensionais ou prototípicas.

Por considerarmos que estes dois aspectos comportam especiais implicações para os procedimentos de avaliação da vinculação do adulto, serão abordados em maior detalhe no tópico seguinte.

\section{A avaliação da vinculação do adulto}

As questões ligadas à avaliação da vinculação do adolescente e do adulto têm ocupado, na actualidade, um grande espaço de debate.

$\mathrm{O}$ interesse e curiosidade que o tema tem suscitado nas últimas décadas têm sido acompanhados de uma proliferação de instrumentos de medida. Estes instrumentos de avaliação têm, muitas vezes, subjacente concepções distintas da vinculação, avaliam diferentes domínios e recorrem a 
abordagens diversas. Uma tal dispersão tem preocupado diversos autores pelo risco de se poder comparar resultados de estudos empíricos obtidos com com base em instrumentos de avaliação bem diferentes. Por outro lado, um tão rápido crescimento tem também criado o receio do risco de distanciamento da teoria original (Hazan \& Shaver, 1994).

Recentemente, temos assistido a esforços que representam tentativas de selecção, ordenação e integração de um conjunto de tópicos sobre a avaliação da vinculação do adulto. De entre as iniciativas, salientamos um conjunto de artigos em forma de debate sobre questões polémicas da área (e.g., Shaver \& Mikulincer, 2002; Waters, Crowell, Elliot, Corcoran \& Treboux, 2002), o desenvolvimento de estudos cujo objectivo é comparar a validade convergente e divergente de dimensões e itens que compõem diversos instrumentos de avaliação (e.g., Fraley, Waller \& Brennan, 2000; Sperling, Foelsch \& Grace, 1996) e, por último, a publicação de obras de referência sobre o tema, nomeadamente, em 1999, a edição do Handbook of Attachment: Theory, research and clinical implications, de Cassidy e Shaver e, mais recentemente, em 2004, o Adult Attachment: Theory, research and clinical implications, de Rholes e Simpson.

Nesta linha, no presente artigo, procuramos analisar o estado da arte, salientando aspectos que consideramos centrais em torno da vinculação do adulto. Começamos por analisar as diferentes abordagens conceptuais subjacentes à avaliação das diferenças individuais, passamos a descrever os domínios temáticos que os diferentes instrumentos pretendem contemplar e, por fim, referimos as metodologias de recolha de informação. Em seguida, centrados na avaliação através de questionários de auto-resposta, focamos aspectos centrais da avaliação através dos questionários de auto-resposta. Por fim, depois de revisto o panorama português sobre a avaliação da vinculação do adulto, passamos a apresentar os estudos psicométricos resultantes da aplicação da Adult Attachment Scale (Collins \& Read, 1990) à população portuguesa.

\section{Abordagens conceptuais de avaliação das diferenças individuais}

Encontramos três grandes tipos de abordagens conceptuais subjacentes ao processo de avaliação da vinculação do adulto: aquelas que se baseiam em concepções categoriais ou tipológicas; as que têm por base concepções dimensionais e, por fim, as que se caracterizam por abordagens prototípicas (Bartholomew \& Shaver, 1998; Matos, 2002).

Como já referimos anteriormente, as abordagens categoriais ou tipológicas encontram as suas origens nos trabalhos de Ainsworth e colaboradores (1978) sobre a avaliação das diferenças individuais na vinculação 
de crianças, através do procedimento laboratorial designado por Situação Estranha.

Posteriormente, Hazan e Shaver (1987), influenciados pelos trabalhos de Ainsworth sobre vinculação durante a infância, procuraram traduzir para a idade adulta, no âmbito da relação amorosa, o mesmo sistema de classificação em três categorias adoptado pela autora. Com este objectivo, construíram um instrumento de auto-resposta, no qual é pedido ao indivíduo que escolha, de entre um conjunto de três parágrafos, descritivos dos três estilos de vinculação (evitante, seguro e ansioso/ambivalente), aquele com que mais se identifica.

É de notar, no entanto, que, com os trabalhos de Hazan e Shaver (1987) e também com Main e colaboradores (George et al., 1984), assistimos a uma mudança do foco de avaliação da vinculação, da organização comportamental da criança, para a organização representacional do adulto.

O instrumento de Hazan e Shaver (1987), embora tenha tido um forte impacto no desenvolvimento da investigação empírica (para uma revisão, cf. Fraley \& Waller, 1998), apresenta limitações (Collins \& Read, 1990; Hazan \& Shaver, 1987; Simpson, 1990), nomeadamente, alguns autores referem que a natureza categorial do instrumento assume que cada estilo é independente dos restantes e que não permite avaliar o grau e extensão em que cada estilo é característico de um indivíduo.

As abordagens dimensionais surgiram numa tentativa de ultrapassar os problemas metodológicos das medidas categoriais. Assim, alguns investigadores começaram a utilizar escalas de avaliação contínuas nos seus instrumentos. Collins e Read (1990) incluem-se neste grupo ${ }^{5}$, procurando identificar e separar as descrições contidas em cada um dos parágrafos do instrumento de Hazan e Shaver (1987), transformando-as em múltiplos itens, que poderiam ser avaliados de forma independente, numa escala de tipo Likert, e que confluiriam em três dimensões: Close, Depend e Anxiety 6.

A possibilidade de o sujeito se situar ao longo de dimensões contínuas possui algumas vantagens: assume uma maior variabilidade entre sujeitos, não impõe fronteiras rígidas de pertença a grupos, exige o esforço conceptual de definição e a operacionalização dos componentes básicos da vinculação, possibilitando, igualmente, estudos psicométricos mais precisos (Fraley \& Waller, 1998; Griffin \& Bartholomew, 1994).

No entanto, dada a perda de informação que uma abordagem puramente dimensional comporta, no sentido de aceder a configurações signifi-

\footnotetext{
${ }^{5}$ A este respeito, é de referir, igualmente, os importantes contributos de Levy e Davis (1988), West e Sheldon (1988) e também de Simpson (1990).

${ }^{6}$ Uma descrição mais detalhada do instrumento será feita mais à frente.
} 
cativas não redutíveis às dimensões (Matos, 2002), muitos dos autores dos instrumentos dimensionais desencorajam explicitamente os investigadores a abandonar os modelos tipológicos (Brennan \& Shaver, 1995; Collins \& Read, 1990; Hazen \& Shaver, 1987).

Como forma de colmatar esta situação, surgem as abordagens prototípicas, que procuram conciliar as abordagens anteriores. Assim, simultaneamente, identificam características de um grupo de sujeitos e assumem a existência de variabilidade individual na pertença ao grupo.

O modelo de Bartholomew (Bartholomew, 1990; Bartholomew \& Horowitz, 1991) é considerado na literatura a referência principal deste tipo de abordagem. De acordo com este modelo, as quatro categorias (seguro, preocupado, desinvestido e amedrontado) localizam-se num espaço bidimensional definido pelo posicionamento positivo ou negativo relativamente ao objecto dos modelos internos dinâmicos (o self e o outro).

No entanto, diversos autores que utilizam escalas dimensionais procuram, através da utilização de procedimentos estatísticos específicos, nomeadamente através da análise de clusters, enquadrar os sujeitos em grupos, de acordo com determinadas configurações de resultados ao longo das dimensões, que habitualmente correspondem aos estilos de vinculação (Collins \& Read, 1990; Collins, 1996; Collins \& Feeney, 2000; Feeney, Noller \& Hanrahan, 1994; Eng, Heimberg, Hart, Schneier \& Liebowitz, 2001). Este procedimento constitui também uma aproximação à abordagem prototípica.

\section{Dimensões temáticas subjacentes à vinculação do adulto}

A avaliação sobre a vinculação do adulto tem-se centrado em três grandes dimensões temáticas: as relações com os pais durante a infância; as relações com figuras de vinculação específicas na adolescência e idade adulta, nomeadamente os pais e os pares; e a vinculação ao companheiro(a). Recentemente, a este propósito, Shaver e Mikulincer (2002) referiram que este facto se prende a aspectos conceptuais relacionados com a existência de múltiplos modelos internos dinâmicos,

that can be conceptualized as hierarchically arranged, running, at the bottom, from episodic memories of interaction with particular relationship partners, trough representations of kinds of attachment relationships (e.g. child-parent; romantic, close friendship, client-therapist), to generic representations of attachment relationships. (p. 243).

Esta concepção tem importantes implicações para a avaliação. Os investigadores devem estar atentos à necessidade de tomar opções sobre 
estes aspectos quando constroem ou utilizam medidas de avaliação à vinculação do adulto.

Em relação aos domínios temáticos que os diversos instrumentos procuram contemplar, podemos distinguir diversos tipos, sobrepostos às três dimensões referidas, nomeadamente, (1) um grupo de instrumentos que pretende avaliar as memórias dos adultos sobre as suas experiências, durante a infância e adolescência, relacionadas com a sua história de vinculação (e.g., Adult Attachment Interview, AAI, George, Kaplan \& Main, 1985; Inventory for Assessing Memories of Parental Rearing Behaviour, EMBU, Perris, von Knorring \& Perris, 1980; Parental Bonding Instrument, PBI, Parker, Tupling \& Brown, 1979; Attachment History Questionnaire, AHQ, Pottharst \& Kessler, 1990); (2) um outro conjunto que tem como objectivo avaliar a qualidade da relação com figuras de vinculação particulares, pais ou pares (e.g., Inventory of Parent and Peer Attachment, IPPA, Armsden \& Greenberg, 1987; Reciprocal Attachment Questionnaire for Adults (West \& Sheldon-Keller, 1994); (3) por fim, um conjunto de medidas que pretende avaliar a vinculação ao companheiro(a) (e.g., Attachment Style Questionnaire, Hazan \& Shaver, 1990; Adult Attachment Scale, Collins \& Read, 1990; Relationship Questionnaire, RQ, Bartholomew \& Horowitz, 1991; Relationship Style Questionnaire, RSQ, Griffin \& B., Bartholomew, 1994; Current Relationship Interview, CRI, Crowell \& Owens, 1996; Adult Attachment Questionnaire, AAQ, Simpson, Rholes \& Nelligan, 1992, Simpson, Rholes \& Philips, 1996; Experiences in Close Relationships Revisited, ECR-R., Brennan, Clark \& Shaver, 1998, 2000).

\section{Metodologias de avaliação da vinculação do adulto: entrevistas ou questionários?}

Como mencionámos anteriormente, na década de 80 , surgiram duas grandes linhas de investigação distintas dedicadas à vinculação do adulto, às quais se juntou um terceiro contributo de diversos autores na construção de instrumentos de avaliação. Uma das linhas, coordenada por M. Main centrou-se nas dimensões representacionais das relações de vinculação com os pais durante a infância, avaliadas através da Adult Attachment Interview (AAI, George, Kaplan \& Main, 1985); a segunda, representada por Hazan e Shaver (1987), que, ao explorar o amor romântico como forma de vinculação, utilizaram um instrumento de auto-resposta, baseado nos padrões de vinculação identificados por Ainsworth para a infância.

Estas duas vias de exploração da vinculação do adulto, focadas em dimensões temáticas da vinculação distintas (uma nas relações parentais e outra nas relações com o companheiro), têm as suas origens em diferentes culturas disciplinares. Main, como discípula de Ainsworth, recebeu forma- 
ção analítica, interessou-se pelos problemas clínicos, trabalhou com pequenos grupos, preferindo, como métodos de avaliação, entrevistas e medidas de observação comportamental, que revelariam de forma indirecta a organização da vinculação, partindo do pressuposto que os indivíduos não estão conscientes dessa dinâmica. Hazan e Shaver, como psicólogos sociais, habituados a pensar em termos de traços de personalidade e interacções sociais, com interesse na população em geral, preferiram estudar amostras maiores, através de questionários de auto-resposta, que partem do pressuposto que os indivíduos têm a capacidade de descrever as suas emoções e comportamentos associados às relações significativas. Cada uma destas equipas concentrou a sua atenção nos seus objectos de estudo e metodologias, não abrindo muitas vias de comunicação entre os seus trabalhos (Bartholomew \& Shaver, 1998).

Como ambas as linhas de investigação cresceram em torno da Teoria da Vinculação de Bowlby e Ainsworth, ambas centradas na avaliação das diferenças individuais, muitos assumiram que as duas classificações seriam equivalentes. Este é um enorme equívoco, por todos os motivos de natureza conceptual que abordámos anteriormente.

Nos últimos anos, surgiram alguns artigos (e.g., Borman \& Cole, 1993; Crowell \& Treboux, 1995) que comparam medidas da vinculação do adulto pertencentes às duas tradições que acabámos de apontar. De forma geral, os autores concluem que as medidas não são equivalentes e habitualmente questionam a validade dos instrumentos de auto-resposta. A este propósito, são esclarecedores na identificação de tópicos que tornam esta comparação inadequada: divergência nas dimensões temáticas da vinculação avaliadas; diferença no conceito de modelo interno dinâmico subjacente (no sentido do exposto anteriormente por nós próprios, a respeito da posição de Shaver e Mikulincer, 2002); e, por último, inadequação dos procedimentos estatísticos utilizados.

Bartholomew e Shaver (1998) concluem que, podendo existir tendência para a convergência dos diversos tipos de medida, quando se trata da avaliação de representações genéricas da vinculação, quando comparados diferentes domínios específicos dos padrões de vinculação, os padrões serão necessariamente diferentes. Parece-nos que esta interpretação sobre a comparação de metodologias é consistente com a ideia central de Bowlby (1988) de que, embora a vinculação do adulto tenha as suas raízes nas interacções com as figuras de vinculação durante a infância, é influenciada, ao longo da vida, por experiências como a morte de uma figura de vinculação, a qualidade de uma relação amorosa ou por uma intervenção psicoterapêutica. Ao longo das trajectórias desenvolvimentais, é possível assistir à divergência de modelos internos dinâmicos das relações com os pais daqueles que se formam baseados nas relações com parceiros românticos ou 
amigos significativos e ao subsequente processo de integração destes modelos, modificando os modelos internos dinâmicos mais genéricos (Bartholomew e Shaver, 1998; Collins \& Read, 1994).

Ao comparar a utilização de entrevistas e questionários na avaliação da vinculação do adulto, Crowell et al. (1999) referem três argumentos a favor da utilização de instrumentos de auto-resposta para avaliar diferenças individuais da vinculação na idade adulta: o facto de a vinculação ter um papel muito importante na vida emocional dos indivíduos (Bowlby, 1973, 1980) e os adultos poderem fornecer informação sobre as suas experiências emocionais 7 ; um segundo argumento diz respeito ao facto de os adultos terem suficiente experiência relacional para saberem descrever como se sentem e comportam nessas relações, bem como conhecer o tipo de afirmações dos parceiros sobre o seu comportamento; por último, o facto de os processos conscientes e inconscientes concorrerem para o mesmo objectivo.

No entanto, Crowell et al. (1999) consideram que os questionários de auto-resposta, sendo úteis para avaliar as diferenças individuais relativamente à segurança, não o são para avaliar a dinâmica existente entre a vinculação e as estratégias defensivas. Por outras palavras,

placing a person in the two-dimensional attachment style space is not, by itself, the same as determining why the person is located in a particular region of the space (Crowell et al., 1999, p. 453).

Ponderando todos estes aspectos, convém mencionar que, a propósito da escolha de instrumentos de avaliação da vinculação do adulto, defendemos a necessidade de opções fundamentadas, baseadas na conceptualização da vinculação subjacente, nas variáveis a estudar e nos objectivos a atingir.

Em Portugal, têm vindo a ser dadas algumas contribuições para avaliar a vinculação na adolescência e idade adulta, que têm permitido a realização de estudos empíricos sobre o tema no nosso país. Nomeadamente, Isabel Soares e a sua equipa têm trabalhado na aplicação da $A A I$ a diversos grupos da nossa população (e.g., Jongenelen, 2003; Machado, Soares \& Silva, 1996; Soares, 1996; Soares, Dias, Fernandes, Klein, Alves, Ferreira, Felgueiras, Pinho, Neves, Figueiredo, Jongenelen, Matos, Gonçalves, Ma-

\footnotetext{
${ }^{7}$ Sobre este tópico, também Bartholomew e Shaver (1998) referiram que muitos dos estudos que, ao encontrar pequena associação entre os questionários e as entrevistas, concluem que os questionários são especialmente vocacionados para medir o erro, não têm em consideração diversos estudos (e.g., Shaver \& Brennan, 1992; Feeney \& Noller, 1991) que revelam elevadas associações entre a vinculação e a observação da comunicação conjugal, a forma de terminar relações ou ainda a forma de procurar e fornecer apoio social em épocas adversas.
} 
chado \& Cunha, 2002); Neves, Soares e Silva (1999) validaram o Inventory of Parent and Peer Attachment (IPPA, que recebeu a designação em Portugal de Inventário da Vinculação na Adolescência) e Paula Mena Matos desenvolveu dois instrumentos de vinculação designados por Questionário de Vinculação ao Pai e à Mãe (QVPM, 2002) e Questionário de Vinculação Amorosa (QVA, 2002).

Também um de nós (Canavarro, 1997) iniciou, no final da década de 90, o processo de validação da Adult Attachement Scale (Collins \& Read, 1990), que em Portugal recebeu o nome de Escala de Vinculação do Adulto (EVA). O robustecimento da amostra da população em geral, assim como a aplicação da escala a grupos clínicos têm vindo a decorrer até ao presente momento, oferecendo dados importantes para o estudo psicométrico deste instrumento de avaliação. O processo e resultados decorrentes destes trabalhos serão descritos a seguir.

\section{A avaliação da vinculação do adulto através de questionários de auto-resposta: o caso da Adult Attachment Scale-R}

\section{Descrição do instrumento original}

A escala foi construída por Collins e Read (e revista, pelos mesmos autores, em 1990) com o objectivo de ultrapassar as limitações inerentes ao instrumento de três itens de Hazan e Shaver (1987), que referimos anteriormente.

Na construção da escala, os autores extraíram, em primeiro lugar, as afirmações dos parágrafos do instrumento de Hazan e Shaver (1987), obtendo quinze itens (cinco para cada estilo de vinculação). Em seguida, seis novos itens foram acrescentados, para incluir dois aspectos fundamentais da vinculação, não incluídos no instrumento de Hazan e Shaver: (a) crenças sobre a disponibilidade da figura de vinculação e a sua resposta quando requerida (três itens) e (b) reacções à separação da figura de vinculação (três itens). A versão preliminar da escala ficou, assim, composta por vinte e um itens, sete para cada estilo de vinculação. Numa amostra de 286 mulheres e 184 homens, foram realizados estudos psicométricos do instrumento dos quais resultou a actual versão de 18 itens.

A análise factorial dos 18 itens revelou a presença de três dimensões, cada uma das quais constituída por seis itens. A primeira, designada por Close, avalia a forma como o indivíduo se sente confortável ao estabelecer relações próximas e íntimas; a segunda, Depend, avalia a forma como os indivíduos sentem poder depender de outros em situações em que necessitam deles; por último, a terceira, Anxiety, avalia o grau em que o indivíduo se sente preocupado com a possibilidade de ser abandonado ou rejeitado. 
Correlações feitas entre os três factores revelaram uma ligação modesta entre os factores Depend e Close $(r=0,41)$, sugerindo que as pessoas que possuem confiança nos outros tendem a sentir-se mais confortáveis com a proximidade emocional; uma ligação fraca entre os factores Anxiety e Depend $(r=-0,18)$ e a inexistência de ligação entre os factores Anxiety e Depend $(r=-0,08)$.

Os autores apontam índices de razoável fiabilidade para a escala, referindo alphas de Cronbach para as três dimensões $(0,75$, para a dimensão Depend; 0,72, para a dimensão Anxiety e 0,69, para a dimensão Close). Collins \& Read (1990) referem, para um período de dois meses, uma estabilidade temporal de $r=0,52$, para a subescala Anxiety, $r=0,68$ para a subescala Close e $r=0,71$ para a subescala Depend.

Aspectos da validade convergente da escala são mencionados pelos mesmos autores, através da tradução das dimensões em estilos de vinculação, feita a partir de procedimentos de análise de funções discriminantes e de análise de clusters, que organizaram os resultados ao longo de três grupos teoricamente consistentes com os três estilos de vinculação referidos por Hazan e Shaver (1987).

Como a validade de um instrumento é um processo cumulativo, sempre em aberto, alguns estudos posteriormente elaborados com a AAS-R merecem também a nossa atenção.

Com base num modelo hipotético de associação entre modelos internos dinâmicos, cognições (atribuições), emoções e comportamentos, Collins (1996), numa amostra de 135 indivíduos, procurou analisar a influência da vinculação (avaliada através da AAS-R) e dos estilos atribucionais nas emoções e comportamentos. Os resultados revelaram que ambas as variáveis consideradas são preditores das componentes comportamentais, mas apenas o estilo de vinculação é capaz de predizer as respostas emocionais. Também neste trabalho Collins utilizou procedimentos de análise de clusters para transformar as dimensões da AAS-R nos estilos de vinculação de Hazan e Shaver (1987).

Posteriormente, em 2000, Collins e Feeney, numa amostra de 93 casais, investigaram a associação entre diferenças individuais de vinculação e capacidade de pedir e fornecer apoio, no contexto de relações íntimas. Neste estudo, salientamos os resultados sobre a importância das diferenças individuais na vinculação (avaliadas por transformação das dimensões da AAS-R nos estilos de vinculação de Hazan e Shaver (1987), através de procedimentos de análises de clusters): a vinculação evitante surge como um bom preditor de ineficácia na procura de suporte e a vinculação ansiosa prediz baixa capacidade para fornecer suporte.

Eng et al. (2001) exploraram a organização da vinculação em indivíduos com ansiedade social. Com este objectivo, numa amostra de 118 pa- 
cientes com ansiedade social, realizaram uma análise de clusters, com base nas dimensões da AAS-R. Estes indivíduos incluem-se preponderantemente nos clusters associados a estilos de vinculação ansioso e seguro. Os indivíduos representados pelo cluster correspondente ao estilo de vinculação ansioso, por comparação com os representados pelo cluster correspondente a vinculação segura, apresentam níveis mais elevados de ansiedade social e evitamento, maiores níveis de depressão e menor satisfação com a vida.

Mallinckrodt, Coble e Gantt (1995) examinaram a relação entre os estilos de vinculação e a aliança terapêutica junto de 76 mulheres acompanhadas em psicoterapia, tendo encontrado uma correlação negativa entre a sub-escala Anxiety e dimensões da aliança terapêutica.

Com o objectivo de explorar a congruência entre diversas medidas de auto-relato da vinculação na idade adulta, Sperling, Foelsch e Grace (1996) administraram o instrumento de Hazan e Shaver para avaliação dos estilos de vinculação (1987), a Attachment Style Measure (Simpson. 1990), a Adult Attachment Scale (Collins \& Read, 1990), o Attachment Style Inventory (Sperling \& Berman, 1991), o Reciprocal Attachment Questionnaire (West, Sheldon \& Reiffer, 1987) e a Anxious Romantic Attachment Scale (Hindy, Schwarz \& Brodsky, 1989) a 160 estudantes universitários. Globalmente, os resultados indicam níveis satisfatórios de consistência interna nos diferentes instrumentos e valores de correlação significativos entre as diversas medidas.

Holtzworth-Munroe, Stuart e Hutchinson (1997) recorreram à AAS, no sentido de avaliar diferenças entre sujeitos violentos e não violentos, em contexto conjugal. Os resultados permitem concluir que os sujeitos violentos apresentam valores superiores na escala Anxiety e valores inferiores nas escalas Close e Depend, relativamente aos dos verificados nos sujeitos não violentos.

\section{Estudos psicométricos realizados em Portugal}

Os primeiros estudos psicométricos resultantes da aplicação da Adult Attachment Scale à população portuguesa foram realizados por um de nós (Canavarro, 1997). Nessa ocasião, à versão portuguesa da escala correspondeu a designação de Escala de Vinculação do Adulto (EVA), designação que se mantém actualmente.

Posteriormente, outros estudos realizados com a EVA, por diversos autores (Tereno, 2001; Almeida, 2005), permitiram robustecer a amostra inicial, oferecendo, assim, importantes contributos para o estudo das qualidades psicométricas da versão portuguesa da escala. 
Nos primeiros estudos psicométricos realizados (Canavarro, 1997), procedeu-se à tradução do instrumento original de Collins e Read (1990, versão revista). Seguidamente, foi feita a retroversão e a passagem do instrumento a um pequeno grupo de pessoas, com o objectivo de verificar a acessibilidade do vocabulário e a compreensão unívoca dos itens, de acordo com os procedimentos sugeridos por De Figueiredo e Lemkau (1980) para estas situações.

Finalmente, a escala foi preenchida por uma amostra de 192 indivíduos (150 mulheres e 42 homens), maioritariamente casados ou a viver maritalmente e com um grau de instrução correspondente a um curso médio ou à frequência universitária.

Nessa ocasião, os estudos da consistência interna da EVA revelaram, através da análise da média dos itens (e dado que o desvio-padrão varia entre 0,731 e 1,155 ) que todos eles se encontram bem centrados. O índice de alpha de Cronbach correspondente a cada item apresentava valores dentro dos intervalos considerados adequados, situando-se entre 0,759 e 0,688. A análise das correlações entre o item e o valor global, e o item e o valor global, excluindo o item, revelou que os itens 1 e 14 não apresentavam valores considerados adequados de acordo com os critérios de Streiner e Norman (1989), ou seja, superiores a 0,2 , o que conduziu à sua posterior reformulação e ajustamento.

No referido estudo, num subgrupo de 102 indivíduos, foi também avaliada a estabilidade temporal do instrumento. $\mathrm{O}$ tempo entre o teste e o reteste correspondeu a um intervalo mínimo de seis semanas. Verificou-se que as correlações encontradas (entre 0,423 e 0,645 ) eram significativas, indicando boa estabilidade temporal da escala.

Com o duplo objectivo de avaliar a validade do instrumento e conhecer as dimensões factoriais subjacentes, procedeu-se à extracção de componentes principais, com rotação de tipo varimax. Os 18 itens foram então submetidos a uma análise factorial, da qual foi possível extrair seis factores ortogonais. Como critério de retenção de componentes, utilizámos o recomendado por Stevens (1996), que se baseia num método gráfico designado por scree-test. Aplicando o critério mencionado, retivemos três factores, equivalentes aos encontrados, quando, na análise factorial exploratória de tipo varimax, pedimos a extracção de três factores. Os factores encontrados nessa ocasião, com capacidade para explicar $46,62 \%$ da variância total, eram condizentes com a Teoria da Vinculação do Adulto, mas, dada a forma como os itens se organizavam, optámos, de forma semelhante ao efectuado posteriormente por Kurdek (2002), por designar as dimensões de acordo com o modelo de Hazan e Shaver (1987), isto é, dimensões de Vinculação Ansiosa, Segura e Evitante. 
Canavarro (1997-1999) refere o poder discriminativo das três dimensões da EVA para distinguir entre população em geral e dois grupos de indivíduos com perturbações emocionais, respectivamente, um com perturbações depressivas e um outro com perturbações mediadas pela ansiedade. Pela sua relação com o presente artigo, salientamos mais alguns resultados do estudo referido. Nomeadamente, ao avaliar, através do EMBU, a influência das relações afectivas estabelecidas com os pais, na infância e adolescência, na vinculação na idade adulta, os resultados sugerem que pais carinhosos, disponíveis, atentos e capazes de responder às necessidades dos filhos (isto é, que os suportam emocionalmente) parecem ter contribuído para a formação de dimensões associadas a vinculação segura na idade adulta. No entanto, é de salientar que

diversos dados confluem no sentido que a maior contribuição para a saúde mental do adulto é oferecida pelas relações afectivas estabelecidas na idade adulta" (Canavarro, 1999, p. 320).

Posteriormente, à amostra utilizada nos primeiros estudos de caracterização psicométrica do instrumento, juntaram-se as amostras da população em geral, utilizadas nos estudos de Tereno (2001) e de Almeida (2005). A amostra total ficou, assim, constituída por 434 sujeitos da população em geral, provenientes das zonas norte e centro do país. A faixa etária compreende o intervalo entre os 18 e os 63 anos $(\mathrm{M}=25 ; D P=8,75)$, sendo $83,2 \%$ dos sujeitos do sexo feminino e $16,8 \%$ do sexo masculino. Em Termos de habilitações literárias, $7,4 \%$ dos sujeitos possuem o ensino básico, 13\% o ensino secundário e $79,6 \%$ frequentam ou concluíram o ensino superior.

Neste segundo estudo, a análise das características psicométricas da escala foi realizada utilizando o programa informático SPSS, versão 13 para Windows (SPSS, 2004), em duas fases distintas.

Num primeiro momento, interessados em reavaliar, em primeiro lugar, a estrutura dimensional da escala, procedeu-se a uma Análise Factorial Exploratória de Componentes Principais (rotação varimax) pedindo a extracção de três factores.

Em seguida, foram realizadas análises sobre a fiabilidade do instrumento, de acordo com a escala como um todo e com as dimensões encontradas.

Os resultados desta análise factorial, quanto à saturação, valor próprio e variância explicada, são apresentados no Quadro 1.

Tal como se verifica, esta solução é responsável por $46,9 \%$ da variância total, sendo o factor 1 constituído por itens que se relacionam com a dimensão Ansiedade, o factor 2 composto por itens que se associam à dimensão de Conforto com a Proximidade e o factor 3 constituído por itens que se relacionam com a dimensão Confiança nos Outros. 
Quadro 1: EVA - solução de 3 factores ortogonais após análise factorial

\begin{tabular}{|c|c|c|c|c|c|}
\hline \multicolumn{2}{|c|}{ Ansiedade } & \multicolumn{2}{|c|}{ Conforto com a Proximidade } & \multicolumn{2}{|c|}{ Confiança nos Outros } \\
\hline Item & Peso & Item & Peso & Item & Peso \\
\hline 9 & 0,784 & 12 & 0,750 & 18 & 0,688 \\
\hline 10 & 0,773 & 1 & 0,715 & 2 & 0,619 \\
\hline 3 & 0,762 & 14 & 0,648 & 16 & 0,539 \\
\hline 11 & 0,754 & 6 & 0,567 & 17 & 0,483 \\
\hline 15 & 0,591 & $8^{*}$ & $-0,396$ & 7 & 0,444 \\
\hline 4 & 0,570 & $13 *$ & $-0,343$ & $5^{*}$ & $-0,312$ \\
\hline \multicolumn{6}{|c|}{ Valor próprio } \\
\hline \multicolumn{2}{|c|}{5,00} & \multicolumn{2}{|c|}{1,93} & \multicolumn{2}{|c|}{1,52} \\
\hline \multicolumn{6}{|c|}{ Variância explicada } \\
\hline \multicolumn{2}{|c|}{$27,73 \%$} & \multicolumn{2}{|c|}{$10,74 \%$} & \multicolumn{2}{|c|}{$8,45 \%$} \\
\hline \multicolumn{6}{|c|}{ Total variância explicada } \\
\hline
\end{tabular}

$46,92 \%$

Nota: * Itens invertidos.

Esta solução factorial replica parcialmente a estrutura proposta por Canavarro (1997), aproximando-se, no entanto, mais da do instrumento original (Collins \& Read, 1990).

Embora exista paralelismo entre as dimensões que anteriormente designámos por Dimensões de Vinculação Ansiosa, Segura e Evitante e as actuais dimensões designadas, respectivamente, por Ansiedade; Conforto com a Proximidade e Segurança nos Outros, como se poderá constatar através da contribuição dos itens para cada dimensão, as alterações verifi$\operatorname{cadas}^{8}$ e a ponderação de alguns aspectos conceptuais levaram-nos a optar pelas designações que indicamos neste trabalho, que reflectem, de forma mais linear, as adoptadas por Collins e Read (1990; 1994).

Assim, o factor 1, Ansiedade, refere-se ao grau de ansiedade sentida pelo indivíduo, relacionada com questões interpessoais de receio de abandono ou de não ser bem querido; o factor 2, Conforto com a Proximidade,

\footnotetext{
${ }^{8}$ Em relação às dimensões encontradas por Canavarro (1997), verifica-se uma distribuição mais equitativa em relação ao número de itens por dimensão (seis), dado que os itens 7 e 5, anteriormente pertencentes à dimensão de Ansiedade, apresentam agora maior peso na dimensão três, que designámos por Confiança nos Outros; por sua vez, o item 13, anteriormente pertencente à dimensão Evitamento, tem, neste trabalho, maior peso na Dimensão Conforto com a Proximidade.
} 
refere-se ao grau em que o indivíduo se sente confortável com a proximidade e a intimidade; e, por último, o factor 3, Confiança nos Outros, diz respeito ao grau de confiança que os sujeitos têm nos outros, assim como na disponibilidade destes quando sentida como necessária.

Em relação à comparação da estrutura da EVA com a AAS-R, verificamos que todos os itens saturam nas mesmas dimensões, com a excepção dos itens 17 e 14. O item 17, em sentido invertido, pertence à dimensão Close na AAS-R e na EVA, pertence à dimensão Confiança nos Outros, sem inversão do sentido; a situação inversa acontece com o item 14. Se atendermos ao conteúdo dos itens e à sua ponderação em sentido directo ou inverso, este facto não interfere com o significado das dimensões nas quais se incluem.

Uma segunda etapa dos estudos psicométricos foi feita no sentido de encontrar indicadores de fiabilidade do instrumento. Com esse objectivo, analisámos as correlações entre o item e o valor das sub-escalas, excluindo o item. Foram igualmente encontrados os valores globais para os coeficientes de Spearman-Brown e de correlação split-half.

Quadro 2: EVA - correlação item-total e alpha se o item for retirado, por sub-escala

\begin{tabular}{lccc}
\hline Factor & Item & R item-total & $\begin{array}{c}\text { Alpha } \\
\text { (sem item) }\end{array}$ \\
\hline & 3 & 0,674 & 0,808 \\
Ansiedade & 4 & 0,495 & 0,841 \\
& 9 & 0,686 & 0,805 \\
& 10 & 0,660 & 0,811 \\
& 11 & 0,686 & 0,806 \\
Conforto com a & 15 & 0,540 & 0,834 \\
Proximidade & 1 & 0,461 & 0,606 \\
& 6 & 0,276 & 0,677 \\
& $8 \mathrm{r}$ & 0,402 & 0,631 \\
& 12 & 0,482 & 0,601 \\
Confiança & $13 \mathrm{r}$ & 0,323 & 0,655 \\
nos Outros & 14 & 0,487 & 0,596 \\
\hline $2 \mathrm{r}$ & 0,211 & 0,535 \\
& 5 & 0,037 & 0,587 \\
& $7 \mathrm{r}$ & 0,311 & 0,484 \\
& $16 \mathrm{r}$ & 0,379 & 0,440 \\
& $17 \mathrm{r}$ & 0,275 & 0,497 \\
& $18 \mathrm{r}$ & 0,513 & 0,369 \\
\hline
\end{tabular}

No Quadro 2, encontram-se, por sub-escala, os valores de correlação item-total e alpha se o item for retirado, de acordo com a estrutura factorial. Numa análise global, é possível verificar que os itens apresentam cor- 
relações satisfatórias com os totais das sub-escalas, exceptuando-se o item 5 na dimensão Confiança. No entanto, dada a importante contribuição que este item apresenta para a dimensão Confiança nos Outros, optámos por o incluir na versão final da escala.

O Quadro 3 apresenta os indicadores de fiabilidade para as sub-escalas da EVA e para o total da escala. A sub-escala Ansiedade apresenta um valor elevado de alpha $(0,84)$, o que não acontece com as sub-escalas Conforto com a Proximidade e sobretudo com a Confiança nos Outros, que apresentam valores de 0,67 e de 0,54 , respectivamente, constituindo valores um pouco inferiores ao desejável.

O valor de alpha para o total da escala é elevado $(0,81)$, registando-se o mesmo para os índices de Spearman-Brown $(0,84)$ e de correlação split-half $(0,83)$.

Quadro 3: EVA - valores de alpha de Cronbach, Spearman-Brown e Guttman Split-half

\begin{tabular}{lc}
\hline Factor & EVA \\
\hline Ansiedade & $\alpha=.84$ \\
Conforto com a Proximidade & $\alpha=.67$ \\
Confiança nos Outros & $\alpha=.54$ \\
Total & $\alpha$ de Cronbach $=0,81$ \\
& Coeficiente de Spearman-Brown $=0,84$ \\
& Correlação split-half $=0,83$ \\
\hline
\end{tabular}

Procurou-se também conhecer as correlações entre as três dimensões da EVA, tendo-se verificado que a escala de Ansiedade se encontra inversamente correlacionada com as escalas de Conforto com a Proximidade e de Confiança nos Outros $(r=-0,353, p<0,001 ; r=0,391, p<0,001$ respectivamente) e que estas duas últimas se encontram positivamente correlacionadas $(r=0,312, p<0,001)$.

Para explorar a validade discriminativa do instrumento, procedeu-se ao estudo das diferenças de médias entre a amostra normativa e uma amostra clínica, agrupando sujeitos provenientes de 3 estudos distintos (Tereno, 2001; Marques, 2004; Coutinho, 2005). No seu conjunto, a amostra clínica ficou composta por 88 sujeitos, com diagnósticos de anorexia nervosa (47 sujeitos), bulimia nervosa ( 25 sujeitos) e perturbações de internalização (16 sujeitos), com idades compreendidas entre os 18 e os 38 anos, sendo $97 \%$ do género feminino e $3 \%$ do género masculino. 
Os resultados revelaram diferenças significativas entre as amostras nas três sub-escalas: nas escalas de Conforto com a Proximidade e de Confiança nos Outros, os sujeitos da amostra normativa apresentam valores mais elevados do que os registados pelos sujeitos da amostra clínica $(t=4,59 ; p<0,001$ e $t=8,66 ; p<0,001$, respectivamente). Pelo contrário, na sub-escala de Ansiedade, os sujeitos da amostra clínica apresentam valores significativamente mais elevados do que os da amostra normativa $(t=-10,16 ; p>0,01)$.

\section{Resultados da estatística descritiva para as pontuações da escala}

Apresentam-se de seguida os valores de média e desvio-padrão das três sub-escalas para o total da amostra normativa $(\mathrm{N}=434)$.

Dado que o sexo não altera significativamente as pontuações obtidas, não são apresentados os resultados em função do sexo.

Quadro 4: EVA - estatística descritiva para as pontuações das três dimensões

\begin{tabular}{lcc}
\hline & Média & DP \\
\hline Ansiedade & 2,43 & 0,74 \\
Conforto com a Proximidade & 3,49 & 0,58 \\
Confiança nos Outros & 3,27 & 0,53 \\
\hline
\end{tabular}

\section{Procedimentos de análise de clusters: estudo exploratório}

Embora, como mencionámos anteriormente, as abordagens dimensionais proporcionem uma análise mais detalhada da vinculação do adulto, conceptualmente continua a ser útil não perder a possibilidade de aceder a configurações significativas da vinculação, habitualmente correspondentes a estilos de vinculação.

Com este objectivo, utilizando a amostra da população em geral, procedemos a uma análise de clusters utilizando as dimensões Ansiedade, Conforto com a Proximidade e Confiança nos Outros, para classificar os indivíduos em estilos de vinculação, no sentido do já efectuado por diversos autores (Collins \& Read, 1990; Collins, 1996; Collins \& Feeney, 2000; Feeney, Noller \& Hanrahan, 1994; Eng et al., 2001).

Utilizámos o software SPSS (versão 13, 2004) para realizar a análise de clusters, recorrendo ao método não hierárquico $K$-means (Johnson \& Wichern, 2002, in Maroco, 2003). Seguimos os procedimentos recomendados por Collins e Read (1990), tendo igualmente em consideração o referido a este respeito por Collins (1996), para determinar a solução final de 
clusters, o que, na nossa amostra, resultou na opção por uma divisão em três perfis $(k=3)$.

Como se pode observar no Quadro 5, os clusters encontrados correspondem à descrição teórica dos três estilos de vinculação de Hazan e Shaver (1987). O grupo correspondente ao perfil Seguro sente-se confortável com a proximidade, é capaz de confiar nos outros e não sente especial receio de ser abandonado; o grupo correspondente ao perfil Evitante não se sente confortável com a proximidade, não confia nos outros e não sente receio especial em ser abandonado; por último, o grupo correspondente ao perfil Preocupado não se sente confortável com a proximidade, não confia nos outros e sente um grande receio com a possibilidade de ser abandonado. Do total da amostra, 46\% dos indivíduos foram classificados como seguros, $35 \%$ como evitantes e $19 \%$ como preocupados. Estas percentagens correspondem, de forma aproximada, aos estudos prévios que utilizaram esta metodologia.

Quadro 5: Médias das dimensões da EVA para estilos de vinculação obtidos através de uma análise de clusters

\begin{tabular}{|c|c|c|c|c|}
\hline \multirow[b]{2}{*}{ Dimensões EVA } & \multicolumn{4}{|c|}{ Clusters/Estilos de Vinculação } \\
\hline & $\begin{array}{c}\text { Preocupado } \\
(\mathrm{N}=83)\end{array}$ & $\begin{array}{l}\text { Evitante } \\
(\mathrm{N}=150)\end{array}$ & $\begin{array}{c}\text { Seguro } \\
(\mathrm{N}=201)\end{array}$ & $F(2,431)$ \\
\hline $\begin{array}{l}\text { Conforto com a } \\
\text { Proximidade }\end{array}$ & 3,16 & 3,28 & 3,95 & $109,01 * * *$ \\
\hline $\begin{array}{l}\text { Confiança nos Ou- } \\
\text { tros }\end{array}$ & 2,88 & 3,15 & 3,66 & $99,287 * * *$ \\
\hline Ansiedade & 3,54 & 2,44 & 1,80 & $462,944 * * *$ \\
\hline
\end{tabular}

Nota: ${ }^{* * *} \mathrm{p}<0,001$

Uma análise de variância multivariada (MANOVA) dos perfis de vinculação, revelou um efeito estatisticamente significativo, mostrando que a Ansiedade é a dimensão que tem um maior peso discriminativo no estabelecimento dos clusters. As médias de cada dimensão, correspondentes aos estilos referidos, encontram-se igualmente no Quadro 5.

Classificação em protótipos de vinculação a partir da AAS-R: análise exploratória

Partindo da sugestão de N. Collins (1996, comunicação pessoal), procedeu-se também à exploração da classificação dos indivíduos nos qua- 
tro protótipos de vinculação propostos por K. Bartholomew (1990), na amostra normativa.

Com esse objectivo, foram replicados os procedimentos e utilizada a sintaxe construída para este fim, de acordo com as indicações de N. Collins (1996, comunicação pessoal). Assim, primeiro foram calculados os valores médios nas dimensões Ansiedade, Confiança e Conforto com a Proximida$d e$. De seguida, foi calculado o valor compósito das dimensões Confiança nos Outros e Conforto com a Proximidade (valor médio destas duas dimensões, criando a variável Conforto-Confiança (ClosDep, no original). Finalmente, procedeu-se à classificação dos indivíduos a partir dos valores obtidos na variável Conforto-Confiança e na variável Ansiedade do seguinte modo: os indivíduos que apresentassem valores superiores ao valor médio da escala (3) na variável Conforto-Confiança e valores inferiores ao valor médio na variável Ansiedade eram classificados como Seguros; os que apresentassem valores superiores a 3 na variável Conforto-Confiança e superiores a 3 na variável Ansiedade eram classificados como Preocupados; os que apresentassem valores inferiores a 3 na variável Conforto-Confiança e inferiores a 3 na variável Ansiedade eram classificados como Desligados; os que apresentassem valores inferiores a 3 na variável Conforto-Confiança e superiores a 3 na variável Ansiedade eram classificados como Amedrontados.

Como pode ser verificado no Quadro 6, utilizando este procedimento foi possível classificar $88,9 \%$ dos sujeitos, ficando por classificar 48 sujeitos, que apresentaram valores intermédios, ou seja, podem situar-se na fronteira entre vários estilos em simultâneo. A classificação dos sujeitos pelos estilos foi a seguinte: $64,3 \%$ seguros, $10,6 \%$ preocupados, $7,8 \%$ desligados e $6,2 \%$ amedrontados.

Quadro 6: Distribuição dos sujeitos por protótipos de vinculação (Bartholomew, 1990)

\begin{tabular}{lcc}
\hline Estilos (Bartholomew, 1990) & N & \% \\
\hline Seguro & 279 & 64,3 \\
Preocupado & 46 & 10,6 \\
Desligado & 34 & 7,8 \\
Amedrontado & 27 & 6,2 \\
Não classificáveis & 48 & 11,1 \\
\hline Total & $\mathbf{4 3 4}$ & $\mathbf{1 0 0}$ \\
\hline
\end{tabular}




\section{Procedimentos de cotação}

Para obter as pontuações nas três dimensões da EVA, os itens da escala devem ser cotados de 1 a 5 , tendo em atenção que os itens assinalados no Quadro 7 com um asterisco devem ser cotados de forma inversa, por se encontrarem invertidos ${ }^{9}$. Após a cotação dos itens, deverá ser efectuada a soma do conjunto de itens que compõe cada dimensão, dividindo a pontuação obtida pelo número de itens (6).

Quadro 7: EVA - itens pertencentes a cada dimensão (procedimentos de cotação)

\begin{tabular}{lcccccc}
\hline Dimensões & \multicolumn{7}{c}{ Itens } \\
\hline Ansiedade & 9 & 10 & 3 & 11 & 15 & 4 \\
Conforto com a proximidade & 12 & 1 & 14 & 6 & $8^{*}$ & $13^{*}$ \\
Confiança nos outros & $18^{*}$ & $2^{*}$ & $16^{*}$ & $17^{*}$ & $7^{*}$ & 5 \\
\hline
\end{tabular}

Nota: *Itens invertidos

\section{Comentário final}

Os estudos realizados com a EVA possibilitam-nos afirmar que as características psicométricas deste instrumento permitem a sua utilização científica e clínica.

Os resultados no âmbito da fiabilidade e os relativos à validade são bastante bons, à excepção da consistência interna das dimensões Conforto com a Proximidade e, sobretudo, da Confiança nos Outros, que, quando avaliadas através do indicador alpha de Cronbach, apresentam valores um pouco inferiores ao desejável. No entanto, estas subescalas diferenciam população em geral de população clínica e mostram-se bons elementos na construção de estilos de vinculação consistentes com a Teoria da Vinculação do Adulto (Bartholomew, 1990; Collins \& Read, 1990, 1996, 2000; Hazan \& Shaver, 1987), sugerindo que a maioria dos seus conteúdos são pertinentes em termos da vinculação do adulto.

Nas diversas análises efectuadas, os resultados obtidos na dimensão Ansiedade mostram-nos a sua centralidade no instrumento, facto consistente com os mais recentes desenvolvimentos da Teoria da Vinculação do Adulto, que a consideram, conjuntamente com a dimensão evitamento,

\footnotetext{
${ }^{9}$ A este propósito, convém anotar que, para efeitos de cotação, com o objectivo de todas as dimensões poderem variar em sentido crescente, os itens da dimensão Confiança nos Outros foram invertidos em relação à solução factorial antes de ser somados, procedimento igualmente efectuado por Collins \& Read (1990).
} 
como um dos melhores discriminadores das diferenças individuais (Brennann et al., 1998; Collins \& Feeney, 2000; Fraley \& Waller, 1998).

As análises exploratórias adicionais demonstraram que as três dimensões encontradas, dependendo dos procedimentos utilizados, definem perfis de vinculação correspondentes aos estilos identificados por Hazan e Shaver (1987) e Bartholomew (1990). Estes resultados são importantes do ponto de vista conceptual. pois revelam-nos que embora os grupos encontrados difiram, de forma consistente, com as concepções teóricas relativas aos estilos de vinculação que lhe estão subjacentes, também nos revelam que três ou quatro perfis de vinculação é um número limitado para descrever as diferenças individuais da vinculação do adulto.

\section{Agradecimento}

Gostaríamos de agradecer aos investigadores Susana Tereno, João Almeida, Teresa Marques e Joana Coutinho, pela amabilidade que tiveram ao facultar o acesso às suas bases de dados. Este facto constituiu uma importante contribuição para o prosseguimento dos estudos psicométricos da EVA.

\section{Bibliografia}

Ainsworth, M. (1972). Attachment and dependency: a comparison. In J. L. Gewirtz (Ed.), Attachment and dependency (pp. 97-137). Washington, D.C.: Winston.

Ainsworth, M. (1991). Attachments and other affectional bonds across the life cycle. In C. M. Parkes, J. Stevenson-Hinde, \& P. Marris (Eds.), Attachment across the life cycle (pp. 33-51). London: Routledge.

Ainsworth, M., Blehar, M., Waters, E., \& Wall, S. (1978). Patterns of attachment: A psychological study of the strange situation. Hillsdale: Erlbaum.

Almeida, J. (2005). Apoio social e morbilidade em estudantes universitários nos períodos de transição: Estudo comparativo da prevalência da ansiedade e da depressão. Monografia de investigação não publicada. Braga: Universidade do Minho.

Armsden, G. C., \& Greenberg, M. T. (1987). The inventory of parents and peer attachment: Relationships to well-being in adolescence. Journal of Youth and Adolescence, 16, 427-454.

Bartholomew, K. \& Shaver, P. R. (1998). Methods of assessing adult attachment: Do they converge? In J. A. Simpson \& W. S. Rholes (Eds.), Attachment theory and close relationships (pp. 25-45). New York: Guildford Press.

Bartholomew, K. (1990). Avoidance of intimacy: an attachment perspective. Journal of Social and Personal Relationships, 7, 147-178. 
Bartholomew, K., \& Horowitz, L. (1991). Attachment styles among young adults: A test of a four category model. Journal of Personality and Social Psychology, 61 (2), 226-244.

Bartholomew, K., Cobb, R. J., \& Poole, J. A. (1997). Adult attachment patterns and social support processes. In G. R. Pierce, B. Lakey, I. G. Sarason \& B. R., Sarason (Eds.), Source of social support and personality (pp. 359-377). Plenum Press, New York.

Berman, W. H., \& Sperling, M. B. (1994). The structure and function of adult attachment. In M. B. Sperling \& W. H. Berman (Eds.), Attachment in adults clinical and developmental perspectives (pp.3-28). New York: Guildford Press.

Borman, E., \& Cole, H. (1993). A comparison of three measures of adult attachment. Poster presented at the meeting of the Society for Research in Child Development, New Orleans, LA.

Bowlby, J. (1969/1982). Attachment and loss. Vol. 1: Attachment. New York: Basic Books.

Bowlby, J. (1973). Attachment and loss. Vol. 2: Separation. New York: Basic Books.

Bowlby, J. (1980). Attachment and loss. Vol. 3: Loss. New York: Basic Books.

Brennan, K. A. \& Shaver, P. R. (1995). Dimension of adult attachment, affect regulation, and romantic relationship functioning. Personality and Social Psychology Bulletin, 21, 267-283.

Brennan, K. A., Clark, C. L., \& Shaver, P. R. (1998). Self-report measurement of adult attachment: An integrative overview. In J. A. Simpson \& W. S. Rholes (Eds.), Attachment theory and close relationships (pp. 46-76). New York: Guildford Press.

Bretherton, I. (1985). Attachment theory: Retrospect and prospect. In I. Bretherton $\&$ E. Waters (Eds.), Growing points of attachment theory and research. Monographs of the Society for Research in Child Development (pp. 3-35). n. ${ }^{\text {os }} 1-2$, serial n. ${ }^{\circ} 209$.

Canavarro, M. C. (1997). Relações afectivas ao longo do ciclo de vida e saúde mental. Dissertação de doutoramento em Psicologia Clínica apresentada à Faculdade de Psicologia e Ciências da Educação da Universidade de Coimbra. Coimbra, Portugal.

Canavarro, M. C. (1999). Relações afectivas e saúde mental. Coimbra: Quarteto Editora.

Cassidy, J., \& Shaver, P. (1999), (Eds.), Handbook of attachment: Theory, research and clinical implication. NY: Guilford Press.

Collins, N. L. \& Feeney, B. C. (2000). A safe heaven: An attachment theory perspective on support seeking and caregiving in intimate relationships. Journal of Personality and Social Psychology, 78 (6), 1053-1073.

Collins, N. L. (1996). Working models of attachment: Implications for explanation, emotion and behaviour. Journal of Personality and Social Psychology, 71, 810-832. 
Collins, N., \& Read, S. (1990). Adult attachment relationships, working models and relationship quality in dating couples. Journal of Personality and Social Psychology, 58, 644-683.

Collins, N., \& Read, S. (1994). Cognitive representations of adult attachment: The structure and function of working models. In K. Bartholomew \& D. Perlman (Eds.), Advances in personal relationships. Vol. 5: Attachment processes in adulthood (pp. 53-90). London: Jessica-Kingsley.

Coutinho, J. (2005). Influência da qualidade de vinculação do cliente no estabelecimento da aliança terapêutica inicial. Monografia de investigação não publicada. Braga: Universidade do Minho.

Crowell, J. \& Treboux, D. (1995). A review of adult attachment measures: Implications for theory and research. Social Development, 4, 294-327.

Crowell, J., Fraley, R. C., \& Shaver, P. R. (1999). Measurement of individual differences in adolescent and adult attachment. In J. Cassidy \& P. R. Shaver (Eds.), Handbook of attachment: Theory, research and clinical implications (pp. 434-465). New York: Guildford Press.

De Figueiredo, J. M., \& Lemkau, P. V. (1980). Psychiatric interviewing across cultures: some problems and prospects. Social Psychiatry, 15, 117-121.

Dozier, M. (1990). Attachment organization and treatment use for adults with serious psychopahological disorders. Development and Psychopathology, 2, 47-60.

Eng, W., Heimberg, R. G., Hart, T. A., Schneier, F. R., \& Liebowitz, M. R. (2001). Attachment in individuals with social anxiety disorder: The relationship among adult attachment styles, social anxiety, and depression. Emotion, 1 (4), 365-380 .

Feeney, J. A., \& Noller, P. (1991). Attachment style and verbal descriptions of romantic patterns. Journal of Social and Personal Relationships, 8, 187-215.

Feeney, J., Noller, P., \& Hanrahan, M. (1994). Assessing adult attachment. In M. B. Sperling \& W. H. Berman (Eds.), Attachment in adults: Clinical and developmental perspectives (pp. 128-152). New York: Guilford Press.

Fraley, R. C., \& Shaver, P. R. (1998). Airport separation: a naturalistic study of adult attachment dynamics in separation couples. Journal of Personality and Social Psychology, 75, 1198-1212.

Fraley, R. C., \& Waller, N. G. (1998). Adult attachment patterns: A test of the typological model. In J. A. Simpson \& W. S. Rholes (Eds.), Attachment theory and close relationships (pp. 77-114). New York: Guildford Press.

Fraley, R. C. (1999). Attachment continuity from infancy to adulthood: Meta-analysis and dynamic modeling of developmental mechanisms. Manuscript submitted for publication.

Fraley, Waller, \& Brennan (2000). An Item Response Theory Analysis of Self-Report Measures of Adult Attachment. Journal of Personality and Social Psychology, 78 (2), 350-365.

George, C., Kaplan, N., \& Main, M. (1985) The adult attachment interview. Unpublished manuscript, University of California, Berkeley. 
George, C., Kaplan, N., \& Main, M. (1984). Attachment interview for adults. Unpublished manuscript, University of California, Berkeley.

Griffin, D., \& Bartholomew, K. (1994). Models of the self and other: Fundamental dimensions underlying measures of adult attachment. Journal of Personality and Social Psychology, 67 (3), 430-445.

Hazan, C., \& Shaver, P. (1987). Romantic love conceptualized as an attachment process. Journal of Personality and Social Psychology, 52 (3), 511-524.

Hazan, C., \& Shaver, P. (1990). Love and work: An attachment-theoretical perspective. Journal of Personality and Social Psychology, 59, 270-280.

Hazan, C., \& Shaver, P. (1994). Attachment as an organizational framework for research on close relationships. Psychological Inquiry: An International Journal of Peer Commentary and Review.

Hinde, R. A. (1997). Relationships: a dialectical perspective. London: Lawrence Earlbaum Associates.

Hinde, R. A., \& Stevenson-Hinde, J. (1986). Relating childhood relationships to individual characteristics. In W. W. Hartup \& Z. Rubin (Eds.), Relationships and development (pp. 27-50). Hillsdale, NJ: Lawrence Earlbaum Associates.

Holtzworth-Munroe, A., Stuart, G. L., \& Hutchinson, G. (1997). Violent versus non-violent husbands: Differences in attachment patterns, dependence and jealousy. Journal of Family Psychology, 11 (3), 314-331.

Howes, C. (1999). Attachment relationships in the context of multiple caregivers. In J. Cassidy \& P. R. Shaver (Eds.). Handbook of attachment. Theory, research and clinical applications (pp. 671-687). New York: Guilford Press.

Jongenelen, I. (2003). Vinculação em mães adolescentes e seus bebés: da matriz relacional à matriz contextual. Dissertação de doutoramento não publicada. Braga: Universidade do Minho.

Kurdek, L. A. (2002). On being insecure about the assessment of attachment styles. Journal of Social and Personal Relationships, 19, 811-834.

Levy, M. B., \& Davis, K. B. (1988). Lovestyles and attachment styles compared: Their relations to each other and to various relationship characteristics. Journal of Social and Personal Relationships, 5, 439-471.

Lewis, M. (1994). Does attachment imply a relationship or a multiple relationships? Psychological Inquiry, 5, 47-51

Machado, G., Soares, I., \& Silva, C. (1996). Avaliação da representação da vinculação e da percepção da qualidade da relação actual pais-adolescentes. In L. Almeida, S. Araújo, M. Gonçalves, C. Machado \& M. Simões (Org.), Avaliação Psicológica: Formas e Contextos, IV vol. Braga: APPORT.

Main, M., Kaplan, N., \& Cassidy, J. (1985). Security of infancy, childhood, and adulthood: A move to the level of representation. In I. Bretherton \& E. Waters (Eds.), Growing points of attachment theory and research (pp. 66-106). Chicago: University of Chicago Press.

Mallinckrodt, B., Cocle, H. M., \& Gantt, D. L. (1995). Working alliance, attachment memories, and social competencies of woman in brief therapy. Journal of Counseling Psychology, 42 (1), 79-84.

Maroco, J. (2003). Análise estatística com utilização do SPSS. Lisboa: Sílabo. 
Marques, T. (2004). Anorexia Nervosa e Vinculação. Monografia de investigação não publicada. Maia: ISMAI.

Matos, P. M. (2002). (Des)continuidades na vinculação aos pais e ao par amoroso em adolescents. Dissertação de doutoramento, não publicada, apresentada à Universidade do Porto.

Neves, L., Soares, I., \& Silva, M. C. (1999). Inventário da vinculação na adolescência - IPPA. In M. R. Simões, M. M. Gonçalves \& L. S. Almeida (Eds.). Testes e provas psicológicas em Portugal, vol. 2 (pp. 37-48). Braga: APPORT/SHO.

Parker, G., Tupling, H., \& Brown, L. B. (1979). A parental bonding instrument. British Journal of Medical Psychology, 52, 1-10.

Perris, C, Jacobson, L., Lindstorm, H. von Knorring, L. \& Perris, H. (1980). Development a new inventory for assessing memories of parental rearing behaviour. Acta Psychiatrica Scandinava, 61, 265-274

Rutter, M. (1995). Clinical implications of attachment concepts: retrospect and prospect. Journal of Child Psychology and Psychiatry, 36, 549-571.

Scharfe, E., \& Bartholomew, K. (1994). Reliability and stability of adult attachment patterns. Personal relationships, 1, 23-43.

Shaver, P. R. \& Mikulincer, M. (2002). Dialogue on adult attachment: Diversity and integration. Attachment and Human Development, 4 (2), 243-257.

Shaver, P. R., Hazan, C., \& Bradshaw, D. (1988). Love as attachment: The integration of three behavioral systems. In J. Sternberg \& M. L. Barnes (Eds.), The psychology of love (pp. 68-99). New Haven, CT: Yale University Press.

Shaver, P., \& Brennan, K. (1992). Attachment styles and the "big five" personality traits: Their connections with each other and with romantic relationship outcomes. Personality and Social Psychology Bulletin, 18 (5), 536-545.

Simpson, J. (1990). Influence of attachment styles on romantic relationships. Journal of Personality and Social Psychology, 59, 971-980.

Simpson, J. A., Rholes, W. S., \& Nelligan, J. (1992). Support seeking and support giving within couples in an anxiety provoking situation: The role of attachment styles. Journal of Personality and Social Psychology, 62, 434-446.

Simpson, J. A., Rholes, W. S., \& Philips, D. (1996). Conflict in close relationships: An attachment perspective. Journal of Personality and Social Psychology, 71, 899-914

Soares, I. (1996). Representação da vinculação na idade adulta e na adolescência. Estudo intergeracional: mãe-filho(a). Braga: Centro de Estudos em Educação e Psicologia, Instituto de Educação e Psicologia, Universidade do Minho ( $2^{\mathrm{a}}$ edição em 2000).

Sperling, M., Foelsch, P., \& Grace, C. (1996). Measuring adult attachment: Are self-report instruments congruent? Journal of personality assessment, 67 (11), 37 -51 .

SPSS (2004). Statistical Package for the Social Sciences (Version 13.0). Chicago, IL: Author

Stevens, J. (1996). Applied multivariate statistics for the social sciences. Mahwah: Lawrence Earlbaum Associates. 
Streiner, D. L., \& Norman, G. R. (1989). Health Measurement Scales; A practical guide to their development and use. Oxford: Oxford University Press.

Tereno, S., Soares, I., Bouça, D., \& Sampaio, D. (2001). Attachment, family dynamics and therapeutic processes in eating disorders: Preliminary data. The 5 th $^{\text {th }}$ London International Conference on Eating Disorders. Londres (poster).

van IJzendoorn, M. H. (1995). Adult attachment representations, parental responsiveness, and infant attachment: A recta-analysis on the predictive validity of the adult attachment interview. Psychological Bulletin, 117, 387-403.

Waters, E., Crowell, J., Elliot, M., Corcoran, D., \& Treboux, D. (2002). Bowlby’s secure base theory and the social/personality psychology of attachment styles: work(s) in progress [A commentary on Shaver \& Mikulincer's Attachment-related psychodynamics]. Attachment and Human Development, 4, 230-242.

Weiss, R. S. (1982). Attachment in adult life. In C. M. Parkes \& J. Stevenson-Hinde (Eds.), The place of attachment in human behavior (pp. 171-184). New York: Basic Books.

Weiss, R. S. (1991). The attachment bond in childhood and adulthood. In P. Marris, J. Stevenson-Hinde \& C. Parkes (Eds.), Attachment across the life cycle (pp. 66-76). New York: Routledge.

West, M. L., \& Sheldon, A. E. (1988). Classification of pathological attachment patterns in adults. Journal of Personality Disorders, 2, 153-159.

West, M. L., \& Sheldon-Keller, A. E. (1994). Patterns of relating - an adult attachment perspective (pp. 1-23; 53-65). New York: Guildford Press.

West, M. L., \& Sheldon-Keller, A. E. (1994). Psychotherapy strategies for insecure attachment in personality disorders. In M. B. Sperling \& W. H. Berman (Eds.), Attachment in adults - clinical and developmental perspectives (pp. 313-329). New York: Guildford Press. 